\title{
Prevalence of antibodies against Ehrlichia spp. and Orientia tsutsugamushi in small mammals around harbors in Taiwan
}

Kun-Hsien Tsai ${ }^{12^{* *}}$, Shu-Feng Chang ${ }^{3+}$, Tsai-Ying Yen ${ }^{4}$, Wei-Liang Shih ${ }^{4,5}$, Wan-Jen Chen ${ }^{5}$, Hsi-Chieh Wang ${ }^{3}$, Xue-Jie Yu ${ }^{6}$, Tzai-Hung Wen ${ }^{7}$, Wen-Jer Wu ${ }^{8}$ and Pei-Yun Shu ${ }^{3^{*}}$

\begin{abstract}
Background: Tick-borne ehrlichiosis and mite-borne scrub typhus represent important emerging zoonotic rickettsial diseases. Although scrub typhus has been recognized by the Taiwanese public health system, information on ehrlichial infections is scarce in Taiwan. In this study, the risk of spread of ectoparasites on rodents through aerial and marine transportation was assessed in international and domestic harbors. Here, we report the first systematic surveillance of seroprevalence against Ehrlichia spp. in small mammals on the main island of Taiwan.

Methods: In total, 1648 small mammals were trapped from 8 international ports, 18 domestic fishing harbors, and 7 local public health centers around Taiwan from November 2004 to December 2008. Sera were analyzed using indirect immunofluorescence assays to detect lgG antibodies against Ehrlichia chaffeensis and Orientia tsutsugamushi. A serum titer of $\geqq 1: 80$ was considered positive.

Results: Antibodies against Ehrlichia spp. and O. tsutsugamushi were detected in $3.28 \%$ and $4.92 \%$ of small mammals active around harbors, respectively. The seropositive rate against Ehrlichia was higher in northern Taiwan from 2005 to 2008. However, O. tsutsugamushi infections increased in southern Taiwan during this period. The serological evidence of ehrlichial and O. tsutsugamushi infections in all international ports were included in the study. No significant differences were found among the seropositive rates of Ehrlichia spp. and O. tsutsugamushi in small mammals trapped between international and local harbors.

Conclusions: The overall prevalence of Ehrlichia spp. and O. tsutsugamushi infections in small mammals active around harbors was $3.28 \%$ and $4.92 \%$, respectively. The results provided serological evidence supporting the potential risks of transporting pathogens through air and maritime traffic. This study highlights serious issues of the emergence and spread of rickettsial diseases in Taiwan. The incidence of human ehrlichiosis requires further investigation.
\end{abstract}

Keywords: Ehrlichia chaffeensis, Orientia tsutsugamushi, Small mammals, Taiwan

\section{Background}

Rickettsial infections are caused by several genera of obligate intracellular Gram-negative bacteria with life cycles that involve vertebrate and invertebrate hosts. Different types of rickettsiosis are widely distributed worldwide, but

\footnotetext{
*Correspondence: kunhtsai@ntu.edu.tw; pyshu@cdc.gov.tw

${ }^{\dagger}$ Equal contributors

'Institute of Environmental Health, College of Public Health, National Taiwan University, No. 17, Xu-Zhou Road, Taipei 10055, Taiwan

${ }^{3}$ Center for Research, Diagnostics and Vaccine Development, Centers for Disease Control, Ministry of Health and Welfare, No.161, Kun-Yang Street, Taipei 11561, Taiwan

Full list of author information is available at the end of the article
}

the vector species and associated human illnesses are distinct depending on geographical locations. Rickettsioses are classically divided into the scrub typhus group, which is transmitted by mites, the spotted fever group, which is transmitted by ticks or mites, and the typhus group, which is primarily transmitted by lice or fleas. In Taiwan, notifiable rickettsial diseases include scrub typhus, epidemic typhus, and murine typhus. No epidemic typhus has been identified in Taiwan since World War II. Murine typhus, caused by Rickettsia typhi, is endemic to Taiwan, with approximately 20-60 cases each year. Scrub typhus group rickettsiosis is the most prevalent infection and causes $300-$ 
500 confirmed cases annually (http://www.cdc.gov.tw/). Scrub typhus is endemic to Asia, northern Australia, and the western Pacific regions [1,2]. Diverse strains of Orientia tsutsugamushi, the etiological agent of scrub typhus, have been isolated from humans and its vectors, larvalstage trombiculid mites (chiggers) [3, 4]. Epidemiological surveys have suggested considerably high prevalence of $O$. tsutsugamushi infections in wild rodents, ranging from $69.1 \%$ to over $90 \%$, on the offshore and main islands of Taiwan $[5,6]$. Human infections of scrub typhus are routinely diagnosed using molecular, serological and pathogen isolation with shell-vial methods performed at the Centers for Disease Control in Taiwan (Taiwan CDC).

Ehrlichia spp., another genus of bacteria responsible for rickettsial diseases in humans and wild or domestic animals, is less recognized in Taiwan. The first human case of ehrlichiosis was documented in the United States of America in 1986. Ehrlichiosis has since been reported worldwide [7]. The common pathogens that cause human infections are Ehrlichia chaffeensis and E. ewingii. The symptoms are moderate to severe and occasionally are fatal $[8,9]$. Enzootic cycles of Ehrlichia spp. transmitted between ticks and wild animals are widespread and have been reported in Korea, Japan, and southern China, which are in geographic proximity to Taiwan $[10,11]$. Previous studies have identified E. chaffeensis in Rhipicephalus haemaphysaloides and Ixodes granulatus ticks and rodents in Kinmen, one of Taiwan's offshore islands [12]. Here, we report ehrlichial infections in small mammal reservoirs on the main island. The potential impact of human ehrlichiosis in Taiwan has yet to be determined.

The impact of travel on the spread of infectious diseases is of great concern. Human migration has provided a shortcut for disease dissemination. When people travel, they often carry luggage, food and goods, and they are also accompanied by, as their biological microenvironments, microbes, animals, and parasites. To examine the risks arising from transportation of pathogenic Orientia and Ehrlichia through human activities, sera were collected from small mammals captured around harbors, and the prevalence of seropositivity against $O$. tsutsugamush $i$ and Ehrlichia spp. was determined by immunofluorescence assay (IFA). To the best of our knowledge, this is the first systematic surveillance of ehrlichial and Orientia tsutsugamushi infections in their reservoirs on the main island of Taiwan.

\section{Methods}

\section{Study area and small mammal capture}

This study was conducted as part of the surveillance of the Hanta virus in rodents in Taiwan [13]. Small mammals were captured from 33 study sites, including 8 international harbors, 18 domestic fishing harbors, and 7 local public health centers around Taiwan from November
2004 to December 2008 (Fig. 1 and Table 1). The methods for small mammal capture have been described in a previous report [14]. Briefly, approximately 20-30 traps were set up each month in areas with suspected rodent activities. Trapping at international ports took place for three days each month. Sweet potatoes, peanuts and/or sausages were used as bait to attract diverse species of small mammals. Blood was collected by cardiac puncture after the captured animals were anesthetized with Zoletil 50 (Fa. Virbac, Carros, France) and was left to stand for $1 \mathrm{~h}$ at room temperature. Sera were separated by centrifugation, aliquoted, and kept frozen at $-20{ }^{\circ} \mathrm{C}$ for later analysis.

\section{Ethical considerations}

The study was conducted following the regulations of Article 17 of Taiwan Wildlife Conservation Act and with the permission of Taiwan Centers for Disease Control. None of the trapped mammals belonged to Protected Species. All trapping and sampling procedures were performed according to U.S. CDC guidelines for sampling small mammals for virologic testing and met International Health Regulations (IHR2005). The use of animal sera for antibody detection was approved by National Taiwan University College of Medicine and College of Public Health Institutional Animal Care and Use Committee (IACUC Approval No: 20090296).

\section{Serological analysis}

Rodent serum samples were evaluated for reactivity to $E$. chaffeensis (prepared by Prof. Yu's laboratory, the University of Texas Medical Branch) and O. tsutsugamushi (Karp and Kato strains; prepared by Taiwan CDC) using IFA.

For detecting antibodies specific to Ehrlichia spp., antigen slides were prepared with $E$. chaffeensis Arkansasinfected DH82 cells. Briefly, E. chaffeensis-infected DH82 cells from a $150-\mathrm{cm}^{2}$ flask were collected when $100 \%$ of the cells were infected. The cells were centrifuged at $200 \times \mathrm{g}$ for $10 \mathrm{~min}$. Pellets were resuspended in $10 \mathrm{ml}$ of phosphate-buffered saline (PBS) with $0.1 \%$ bovine albumin. Sodium azide was added to a final concentration of $0.01 \%$ and treated at $4{ }^{\circ} \mathrm{C}$ overnight to inactivate Ehrlichia. Ten microliters of antigen was applied to each well of 12-well slides. The slides were air dried and fixed in acetone for 5 min before immunostaining [15].

For the detection of scrub typhus antibodies, O. tsutsugamushi (Karp strain)-infected L929 cells on chamber slides were fixed and permeabilized with ice-cold acetone/ methanol $(1: 1)$ at $-20^{\circ} \mathrm{C}$ for $10 \mathrm{~min}$. The slides were dried and blocked with PBS containing $1 \%$ goat serum before further immunostaining [16].

Serum samples were centrifuged at $1730 \times \mathrm{g}$ at $4{ }^{\circ} \mathrm{C}$ for $10 \mathrm{~min}$ and diluted to 1:80 using a sterile field diluent 


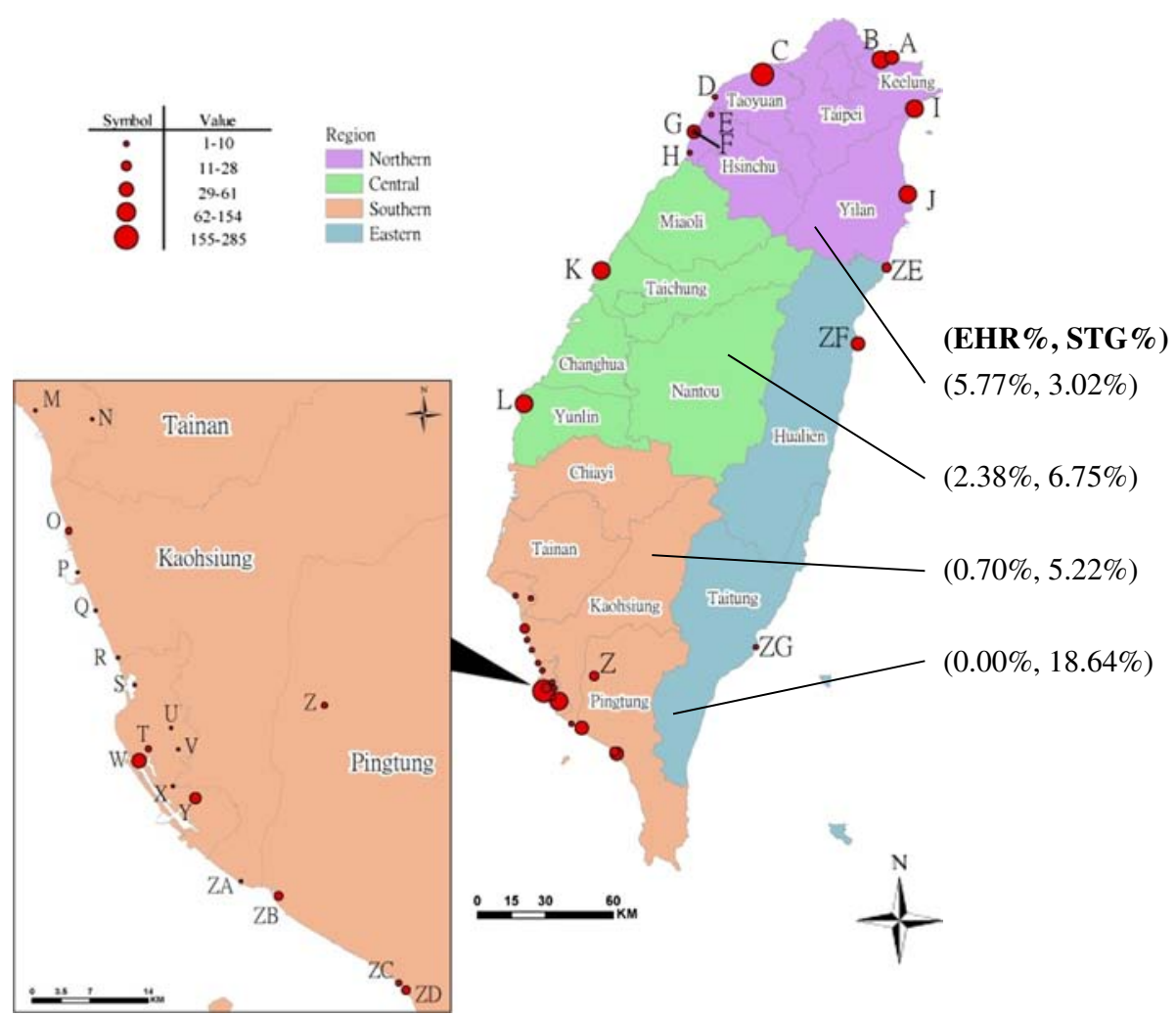

Fig. 1 Thirty-three locations where small mammals were captured around Taiwan. (Purple: northern Taiwan; green: central Taiwan; orange: southern Taiwan; and blue: eastern Taiwan. Value is number of small mammals captured at each site.)

(PBS containing $1.5 \%$ bovine albumin, 100 units $/ \mathrm{mL}$ of penicillin, $100 \mu \mathrm{g} / \mathrm{mL}$ of streptomycin, and $250 \mu \mathrm{g} / \mathrm{mL}$ of amphotericin B). A $20 \mu \mathrm{L}$ volume of rodent serum was pipetted into each well, and the slides were incubated in a moist chamber at $37^{\circ} \mathrm{C}$ for $30 \mathrm{~min}$. The slides were rinsed and washed in PBS for $10 \mathrm{~min}$ and then rinsed again with distilled water. The slides were air-dried, and the wells were incubated with $20 \mu \mathrm{L}$ of fluoresce in isothiocyanate (FITC)-conjugated, affinity-purified, Fc fragment-specific, goat anti-rat IgG (Jackson Immuno-Research Laboratories, West Grove, PA) diluted to 1:100 in PBS containing Evans blue counterstain (Sigma Chemical Company, St. Louis, MO). After incubation in a moist chamber at $37{ }^{\circ} \mathrm{C}$ for $30 \mathrm{~min}$, the slides were rinsed and washed with PBS twice. Coverslips were mounted using mounting medium (PBS:glycerol $=3: 7 \mathrm{v} / \mathrm{v} ; \mathrm{pH}$ 9.0), and the slides were examined at a magnification of $100 \times$ or $400 \times$ with a Zeiss fluorescent microscope. Sera with typical patterns of bright green fluorescence at titers of 1:80 for IgG were deemed positive [17, 18]. Positive and negative control sera were included on each slide. All slides were read independently by two individuals who were blinded to specimen identity. Disagreements were resolved by consensus after re-examining the slides.

\section{Statistical analysis}

All statistical analyses were performed using SAS v9.1.3 software (SAS Institute, Cary, NC). Fisher's exact test was used for comparisons of seroprevalence in rodents between different sampling sites. A twotailed $p$-value of 0.05 or less was considered to be statistically significant. The trend across time was examined with the Cochran-Mantel-Haenszel Chi-squared test.

\section{Results}

\section{Field collection of small mammals}

A total of 1648 small mammals were caught from 33 study sites in Taiwan from November 2004 to December 2008, including 762, 252, 575 and 59 animals in northern, central, southern, and eastern Taiwan, respectively (Table 1). Figure 1 summarizes the numbers of trapped small mammals at the different sites. The seroprevalence of ehrlichial and O. tsutsugamushi infections in 4 districts of Taiwan was determined. Six species of rodents, Rattus norvegicus (brown rat), Bandicota indica (Taiwan bandicoot rat), $R$. losea (brown country rat), Mus musculus (house mouse), $R$. tanezumi (oriental house rat), and Apodemus agrarius (striped field mouse), and a species of insectivore, Suncus murinus (Asian house shrew), 
Table 1 List of the 33 locations around Taiwan where small mammals were captured and rates of seropositivity against Ehrlichia spp. and Orientia tsutsugamushi from November 2004 to December 2008

\begin{tabular}{|c|c|c|c|c|c|c|c|}
\hline \multirow[t]{2}{*}{ Geographic } & \multirow[t]{2}{*}{ Location $\left({ }^{(a)}\right.$} & \multicolumn{6}{|c|}{ Total no. trapped mammals (No. sero-positive to EHR, no. sero-positive to STG) } \\
\hline & & 2004 & 2005 & 2006 & 2007 & 2008 & Total $(2004 \sim 2008)$ \\
\hline \multirow[t]{11}{*}{ Northern } & & 0 & $140(2,2)$ & $186(16,6)$ & $134(12,4)$ & $302(14,11)$ & $762(44,23)$ \\
\hline & A. Badouzih Fishing Harbor (KL) & 0 & 33 & 25 & 0 & 0 & 58 \\
\hline & B. Keelung Harbor $(\mathrm{KL})^{\mathrm{b}}$ & 0 & $44(2,2)$ & $38(3,2)$ & $22(0,1)$ & $30(1,2)$ & $134(6,7)$ \\
\hline & C. Taoyuan Airport (TY) & 0 & 30 & $71(8,2)$ & $80(12,1)$ & $64(2,1)$ & $245(22,4)$ \\
\hline & D. Yongan Fishing Harbor (TY) & 0 & 0 & 0 & 0 & 6 & 6 \\
\hline & E. Potou Fishing Harbor (HC) & 0 & 0 & 1 & 0 & 0 & 1 \\
\hline & F. Nangliao Fishing Harbor (HC) & 0 & 0 & 9 & 0 & 0 & 9 \\
\hline & G. Hsinchu Fishing Harbor ( $\mathrm{HC}$ ) & 0 & 0 & 0 & 0 & $61(2,2)$ & $61(2,2)$ \\
\hline & H. Haishan Fishing Harbor (HC) & 0 & 0 & 0 & 0 & 5 & 5 \\
\hline & I. Dashi Fishing Harbor (YL) & 0 & 0 & 5 & 0 & $84(6,3)$ & $89(6,3)$ \\
\hline & J. Suao Harbor $(Y L)^{b}$ & 0 & 33 & $37(5,2)$ & $32(0,2)$ & $52(3,3)$ & $154(8,7)$ \\
\hline \multirow[t]{4}{*}{ Central } & & 1 & $100(2,6)$ & $66(4,9)$ & 40 & $45(0,2)$ & $252(6,17)$ \\
\hline & K. Taichung Wuchi Harbor (TC) & 0 & 27 & 34 & 22 & 29 & 112 \\
\hline & & & $(0,1)$ & $(1,2)$ & $(0,0)$ & $(0,2)$ & $(1,5)$ \\
\hline & L. Mailiao Harbor $(\mathrm{YL})^{\mathrm{b}}$ & 1 & $73(2,5)$ & $32(3,7)$ & $18(0,0)$ & $16(0,0)$ & $140(5,12)$ \\
\hline \multirow[t]{19}{*}{ Southern } & & 10 & $193(0,4)$ & $130(3,4)$ & $85(0,10)$ & $157(1,12)$ & $575(4,30)$ \\
\hline & M. Anping Fishing Harbor (TN) & 0 & 0 & 2 & 0 & 0 & 2 \\
\hline & N. East District, Tainan city (TN) & 0 & 0 & 2 & 0 & 0 & 2 \\
\hline & O. Hsinda Fishing Harbor $(\mathrm{KH})$ & 0 & 0 & 16 & 3 & 9 & 28 \\
\hline & P. Hsingang Fishing Harbor (KH) & 0 & 0 & 1 & 0 & 0 & 1 \\
\hline & Q. Mito Fishing Harbor (KH) & 0 & 0 & 0 & 0 & 4 & 4 \\
\hline & R. Uhliao Fishing Harbor (KH) & 0 & 0 & 2 & 3 & 5 & 10 \\
\hline & S. Zhoying Military Area (KH) & 0 & 0 & 7 & 0 & 0 & 7 \\
\hline & T. Qianzhen District, Kaohsiung City (KH) & 0 & 0 & 0 & 0 & 15 & 15 \\
\hline & U. Sanmin District, Kaohsiung City (KH) & 0 & 0 & 0 & 0 & 1 & 1 \\
\hline & V. Yanchao District, Kaohsiung City (KH) & 0 & 0 & 4 & 0 & 0 & 4 \\
\hline & W. Kaohsiung International Harbor $(\mathrm{KH})^{\mathrm{b}}$ & 8 & $65(0,2)$ & $51(0,1)$ & $67(0,7)$ & $94(1,9)$ & $285(1,19)$ \\
\hline & X. Chungyun Fishing Harbor (KH) & 0 & 0 & 3 & 0 & 0 & 3 \\
\hline & Y. Kaohsiung International Airport $(\mathrm{KH})^{\mathrm{b}}$ & 2 & 41 & $30(2,3)$ & $12(0,3)$ & $11(0,0)$ & $96(2,6)$ \\
\hline & Z. Yanpu Fishing Harbor (PT) & 0 & 0 & 0 & 0 & $16(0,3)$ & $16(0,3)$ \\
\hline & ZA. Linyuan Fishing Harbor (KH) & 0 & 0 & 0 & 0 & 2 & 2 \\
\hline & ZB. Donggang Fishing Harbor (PT) & 0 & 32 & 6 & 0 & 0 & 38 \\
\hline & ZC. Shueidiliao Fishing Harbor (PT) & 0 & 16 & 0 & 0 & 0 & 16 \\
\hline & ZD. Fangliao Fishing Harbor (PT) & 0 & $39(0,2)$ & $6(1,0)$ & 0 & 0 & $45(1,2)$ \\
\hline \multirow[t]{4}{*}{ Eastern } & & 0 & $6(0,1)$ & $9(0,1)$ & $19(0,2)$ & $25(0,7)$ & $59(0,11)$ \\
\hline & ZE. Hoping Fishing Harbor (HL) & 0 & 0 & 0 & $4(0,2)$ & $16(0,6)$ & $20(0,8)$ \\
\hline & ZF. Hualien Harbor $(\mathrm{HL})^{\mathrm{b}}$ & 0 & $6(0,1)$ & $6(0,1)$ & 15 & $9(0,1)$ & $36(0,3)$ \\
\hline & ZG. Fugang Fishing Harbor (TT) & 0 & 0 & 3 & 0 & 0 & 3 \\
\hline Total & & $11(0,0)$ & $439(4,13)$ & $391(23,20)$ & $278(12,16)$ & $529(15,32)$ & $1648(54,81)$ \\
\hline
\end{tabular}

KL Keelung, TY Taoyuan, HC Hsinhu, TC Taichung, YL Yunlin, TN Tainan, KH Kaohsiung, PT Pingtung, $T T$ Taitung, $H L$ Hualien, YL Yilan 
were identified in this study. The numbers of each species captured and the animals' genders are outlined in Table 2. Rattus norvegicus, S. murinus, and B. indica were the predominant species, accounting for 1102, 284 and 182 of the trapped small mammals, respectively.

\section{Serological analyses of captured animals}

Antibodies against Ehrlichia spp. and O. tsutsugamushi were found in $3.28 \%(54 / 1648)$ and $4.92 \%(81 / 1648)$ of the captured small mammals on the main island of Taiwan, respectively (Table 2). The prevalence was significantly different between ehrlichial (2.99\%; 33/ $1102)$ and $O$. tsutsugamushi infections $(6.26 \% ; 69 / 1102)$ in $R$. norvegicus $(p<0.01)$. The gender of the animals captured appeared to have no influence on the prevalence of both rickettsial infections. Among the rodents, $B$. indica displayed the highest positive rate of $10.44 \%$ (19/182) against Ehrlichia spp., and Ratus tanezumi

Table 2 Species and genders of the captured small mammals and the prevalence of antibodies against Ehrlichia spp. and Orientia tsutsugamushi in small mammals in Taiwan from November 2004 to December 2008

\begin{tabular}{|c|c|c|c|c|c|c|c|}
\hline \multirow[b]{2}{*}{ Species } & \multirow[b]{2}{*}{ Sex } & \multirow[b]{2}{*}{ Total } & \multicolumn{2}{|l|}{ EHR } & \multicolumn{2}{|l|}{ STG } & \multirow[t]{2}{*}{$p$ value } \\
\hline & & & Positive & $\%$ & Positive & $\%$ & \\
\hline \multirow[t]{4}{*}{ Rattus norvegicus } & $M$ & 602 & 17 & 2.82 & 36 & 5.98 & 0.0293 \\
\hline & $\mathrm{F}$ & 489 & 13 & 2.66 & 31 & 6.34 & 0.0244 \\
\hline & U & 11 & 3 & 27.27 & 2 & 18.18 & 1.0000 \\
\hline & total & 1102 & 33 & 2.99 & 69 & 6.26 & 0.0023 \\
\hline \multirow[t]{4}{*}{ Suncus murinus } & M & 102 & 0 & 0.00 & 4 & 3.92 & 0.1213 \\
\hline & $\mathrm{F}$ & 179 & 0 & 0.00 & 0 & 0.00 & - \\
\hline & U & 3 & 0 & 0.00 & 0 & 0.00 & - \\
\hline & total & 284 & 0 & 0.00 & 4 & 1.41 & 0.1237 \\
\hline \multirow[t]{3}{*}{ Bandicota indica } & M & 130 & 13 & 10.00 & 1 & 0.77 & 0.0014 \\
\hline & $\mathrm{F}$ & 52 & 6 & 11.54 & 1 & 1.92 & 0.1123 \\
\hline & total & 182 & 19 & 10.44 & 2 & 1.10 & 0.1886 \\
\hline \multirow[t]{3}{*}{ Rattus losea } & M & 35 & 2 & 5.71 & 1 & 2.86 & 1.0000 \\
\hline & $\mathrm{F}$ & 15 & 0 & 0.00 & 2 & 13.33 & 0.4828 \\
\hline & total & 50 & 2 & 4.00 & 3 & 6.00 & 1.0000 \\
\hline \multirow[t]{3}{*}{ Mus musculus } & M & 6 & 0 & 0.00 & 0 & 0.00 & - \\
\hline & $\mathrm{F}$ & 10 & 0 & 0.00 & 0 & 0.00 & - \\
\hline & total & 16 & 0 & 0.00 & 0 & 0.00 & - \\
\hline \multirow[t]{3}{*}{ Rattus tanezumi } & M & 5 & 0 & 0.00 & 0 & 0.00 & - \\
\hline & $\mathrm{F}$ & 8 & 0 & 0.00 & 3 & 37.50 & 0.2000 \\
\hline & total & 13 & 0 & 0.00 & 3 & 23.08 & 0.2200 \\
\hline \multirow[t]{3}{*}{ Apodemus agrarius } & M & 1 & 0 & 0.00 & 0 & 0.00 & - \\
\hline & $\mathrm{F}$ & 0 & 0 & - & 0 & - & - \\
\hline & total & 1 & 0 & 0.00 & 0 & 0.00 & - \\
\hline Total & & 1648 & 54 & 3.28 & 81 & 4.92 & \\
\hline
\end{tabular}

$M$ male, $F$ female, $U$ unknown exhibited the highest infection rate against $O$. tsutsugamushi (23.08 \%; 3/13).

\section{Seroprevalence in small mammals captured in different geographic locations in Taiwan}

The serological survey revealed that antibodies specific to Ehrlichia spp. were most frequently found in small mammals active around the ports in northern Taiwan (5.77 \%; $44 / 762)$, followed by the central $(2.38 \% ; 6 / 252)$ and southern $(0.70 \% ; 4 / 575)$ districts. The percentage of samples positive for antibodies specific to Ehrlichia spp. was highest in Taoyuan Airport (8.98 \%; 22/245). Furthermore, in agreement with the incidence in human cases, we detected a high prevalence of antibodies against $O$. tsutsugamush $i$ at the Hoping Fishing Harbor (40 \%; 8/20), which is located in eastern Taiwan (Table 1 and Fig. 1).

In this study, 1202 and 446 small mammals were trapped at the international and domestic harbors (including local public health centers), respectively (Table 3 ). The prevalence of antibodies specific to $O$. tsutsugamushi was slightly higher than the prevalence of antibodies specific to Ehrlichia spp. Moreover, a higher but not significantly greater number of animals expressed antibodies against the rickettsial diseases around the international ports. Most importantly, serological evidence of infections was detected in all international harbors (Table 1).

\section{Seroprevalence in small mammals captured at different time points}

The seroprevalence of $O$. tsutsugamushi infections in small mammals increased in the harbors of southern Taiwan during the year of 2004 and $2008(p<0.01)$ (Table 4). Antibodies against Ehrlichia spp. and $O$. tsutsugamushi were detected year-round (Fig. 2). In trapped small mammals, the seropositive rates were highest in November and December for Ehrlichia spp., whereas the positive rates were higher in January and October for O. tsutsugamushi.

Co-infection with different pathogens in captured animals Of the 1648 small mammals tested in the study, 5 (0.30\%) were seropositive against both Ehrlichia spp. and $O$. tsutsugamushi, including one $R$. norvegicus in Keelung Harbor, $2 R$. norvegicus in Mailiao Harbor, one $M$.

Table 3 Comparison of rates of seropositivity against Ehrlichia spp. (EHR), and Orientia tsutsugamushi (STG) infections between international harbors and local harbors in Taiwan

\begin{tabular}{lllllll}
\hline \multirow{2}{*}{ Locations } & & \multicolumn{2}{l}{ EHR } & & \multicolumn{2}{l}{ STG } \\
International Harbor & 1202 & 45 & 3.74 & & 63 & 5.24 \\
Local Harbor & 446 & 9 & 2.02 & & 18 & 4.04 \\
Total & 1648 & 54 & 3.28 & & 81 & 4.92 \\
\hline
\end{tabular}


musculus in Kaohsiung International Airport, and one $R$. norvegicus in Suao Harbor.

\section{Discussion}

In the study, the prevalence of Ehrlichia spp. and $O$. tsutsugamishi infections in captured animals was $3.28 \%$ $(54 / 1648)$ and $4.92 \%(81 / 1648)$, respectively. Most of the seropositive animals were located in northern Taiwan. Serological evidence of infections were present in all international ports. The antibodies were detected in trapped small mammals year-round with an increasing trend in O. tsutsugamushi infections in southern Taiwan from 2004 to 2007. Moreover, this is the first report regarding the prevalence of Ehrlichia spp. infection in small mammals on the main island of Taiwan.

Ehrlichiosis is an important emerging zoonotic disease. Serological studies and molecular detections have associated E. chaffeensis with white-tailed deer, goats, red foxes, coyotes, raccoons, cattle, and Reeves's muntjacs [7, 19-25]. These large wild animals are less common in Taiwan. Conversely, shrews and rodents are more abundant and often serve as reservoirs for many communicable diseases transmitted by their ectoparasites. Previous studies have discovered E. chaffeensis DNA in 16 of 108 $(14.8 \%)$ spleen or liver samples from shrews and rodents on one of Taiwan's offshore islands, Kinmen, by polymerase chain reaction (PCR) and sequencing [14]. Our results further showed that $3.28 \%(54 / 1648)$ of captured animals were seropositive against ehrlichial infections, and more infected animals were found in the north and central parts of the main island of Taiwan. During this study, ectoparasites collected from murine-like animals included two fleas: $X$. cheopis and Nosopsyllus nicanus, three ticks: Ixodes granulatus, Haemaphysalis bandicota, and Rhipicephalus haemaphysaloides, three mites: Laelaps nuttalli, L. sedlaceki, and L. echidninus, and lice. Among these ectoparasites, $45.04 \%$ were X. cheopis, and $29.25 \%$ were $H$. bandicota. In terms of the ectoparasites, $X$. cheopis was the most common species. X. cheopis carries $R$. typhi and serves as a major vector to cause human murine typhus. Haemaphysalis bandicota, the second common ectoparasite, involves in disease transmission among rats. However, $H$. bandicota has never been found to be associated with any vectorborne communicable diseases in humans [26]. Information on trombiculid mites was limited in this study, but these mites may be associated with small mammal species. It is worth noting that serological cross-activities have been addressed among Ehrlichia spp., such as E. chaffeensis, E. ewingii, E. canis and E. ruminantium [27-30]. The enzootic cycle of $E$. canis has been documented in dogs in Taiwan [31, 32]. The positive reactions observed by IFA in the current study therefore may have been confounded by infections with other Ehrlichia species. In North America, the seroprevalence of E. canis, E. chaffeensis and E. ewingii in dogs was $0.8 \%, 2.8 \%$, and $5.1 \%$, respectively [33]. In China, the overall seroprevalence of $E$. chaffeensis was $9.8 \%$ in rural residents and $2.4 \%$ in urban residents [34]. Nevertheless, our results suggested that ehrlichial infections are prevalent on the island. The implications for human ehrlichiosis should be scrutinized and re-evaluated because it is not a commonly observed disease in Taiwan.

Table 4 Trends of rates of seropositivity of antibodies against Ehrlichia spp. (EHR) and Orientia tsutsugamushi (STG) in small mammals in northern, central, southern, and eastern Taiwan from November 2004 to December 2008

\begin{tabular}{|c|c|c|c|c|c|c|c|c|c|c|c|c|}
\hline \multirow[b]{2}{*}{ Variable } & \multicolumn{2}{|c|}{$2004(N=11)$} & \multicolumn{2}{|c|}{$2005(N=439)$} & \multicolumn{2}{|c|}{$2006(N=391)$} & \multicolumn{2}{|c|}{$2007(N=278)$} & \multicolumn{2}{|c|}{$2008(N=529)$} & \multirow[b]{2}{*}{$p$ value } & \multirow{2}{*}{$\begin{array}{l}\text { (trend) } \\
p \text { value }\end{array}$} \\
\hline & $\mathrm{N}$ & $\%$ & $\mathrm{~N}$ & $\%$ & $\mathrm{~N}$ & $\%$ & $\mathrm{~N}$ & $\%$ & $\mathrm{~N}$ & $\%$ & & \\
\hline \multicolumn{13}{|l|}{ EHR } \\
\hline Northern & 0 & 0 & 2 & 0.46 & 16 & 2.81 & 12 & 4.32 & 14 & 0.95 & 0.0054 & 0.8555 \\
\hline Central & 0 & 0 & 2 & 0.46 & 4 & 1.02 & 0 & 0.00 & 0 & 0.00 & 0.1908 & 0.3183 \\
\hline Southern & 0 & 0 & 0 & 0.00 & 3 & 0.77 & 0 & 0.00 & 1 & 0.19 & 0.0845 & 0.8228 \\
\hline Eastern & 0 & 0 & 0 & 0.00 & 0 & 0.00 & 0 & 0.00 & 0 & 0.00 & - & - \\
\hline Total & 0 & 0 & 4 & 0.91 & 23 & 5.88 & 12 & 4.32 & 15 & 2.84 & 0.0003 & 0.3306 \\
\hline$p$ value & & & \multicolumn{2}{|l|}{0.127} & \multicolumn{2}{|l|}{0.115} & \multicolumn{2}{|c|}{0.00007375} & \multicolumn{2}{|c|}{0.040} & & \\
\hline \multicolumn{13}{|l|}{ STG } \\
\hline Northern & 0 & 0 & 2 & 0.46 & 6 & 1.02 & 4 & 0.72 & 11 & 0.95 & 0.9432 & 0.6543 \\
\hline Central & 0 & 0 & 6 & 1.37 & 9 & 2.30 & 0 & 0.00 & 2 & 0.38 & 0.0440 & 0.3680 \\
\hline Southern & 0 & 0 & 4 & 0.91 & 4 & 0.77 & 10 & 3.60 & 12 & 2.27 & 0.0016 & 0.0023 \\
\hline Eastern & 0 & 0 & 1 & 0.23 & 1 & 0.77 & 2 & 1.44 & 7 & 2.46 & 0.7194 & 0.2352 \\
\hline Total & 0 & 0 & 13 & 2.96 & 20 & 4.86 & 16 & 5.76 & 32 & 6.05 & 0.1183 & 0.0264 \\
\hline$p$ value & & & 0.245 & & 0.005 & & 0.00 & & 0.16 & & & \\
\hline
\end{tabular}



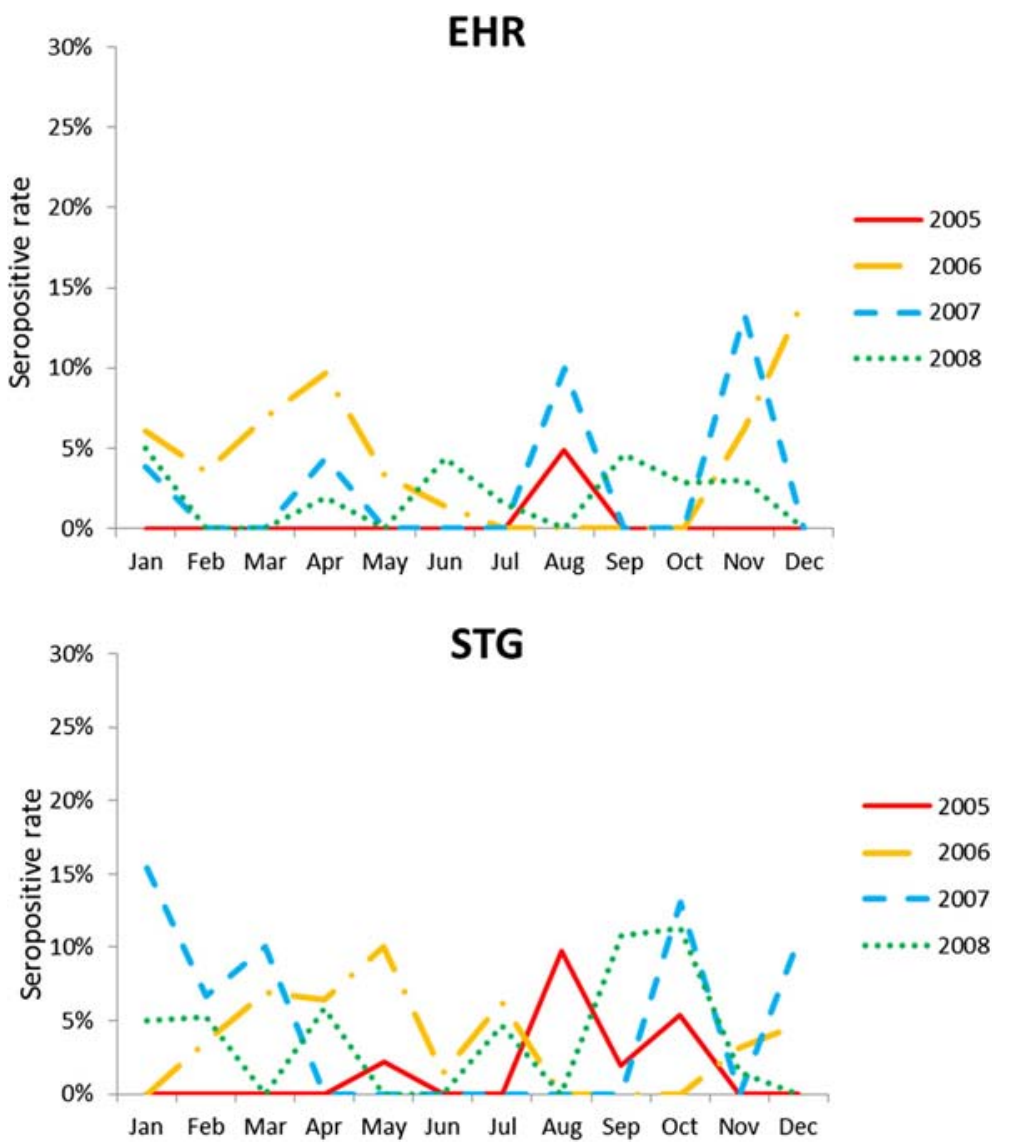

Fig. 2 Seasonal prevalence of antibodies against Ehrlichia spp. (EHR), and Orientia tsutsugamushi (STG) in small mammals from November 2004 to December 2008 in Taiwan

Several studies have evaluated the prevalence of scrub typhus infections in animal reservoirs. A seropositive rate of over $90 \%$ has been reported in rodents in Kinmen [5]. A prevalence of $70 \%$ has been observed in small mammals captured in abandoned agricultural fields in central Hualien County in eastern Taiwan [35]. Our results showed that $4.92 \%$ of the small mammals caught around the harbors expressed antibodies against $O$. tsutsugamushi. The prevalence was relatively low compared with those in previous reports and may reflect the different ecosystems of our sampling areas. Different rodent hosts affect the seropositive rates of rickettsial infections $[5,12,13]$. Our study focused on small mammals active around harbors, which have, to a certain degree, much more human activities than present in the study sites selected in other studies. Furthermore, the rodent species in these harbors differed from those on small islets or in traditional agricultural environments. Indeed, the dominant species among captured animals varies in the literature. $R$. norvegicus was the dominant rodent species in this study, whereas $R$. losea and Apodemus agrarius are abundant in Kinmen and Hualien, respectively $[5,35]$.
The distributions of the rodent species were similar among harbors in Taiwan, with the exceptions of Suao Harbor and Taoyuan Airport. On the whole, $R$. norvegicus was the most frequent species followed by $S$. murinus and B. indica. However, the Taiwan bandicoot rat and brown country rat were the primary rodents captured in Taoyuan Airport. This may be related to the trap sites and the behaviors of $B$. indica, which are usually active in nonresidential areas. Haemaphysalis bandicota was also primarily found at Taoyuan International Airport [26]. For Su-ao Harbor, the only species captured were $R$. norvegicus and M. musculus $(n=1)$.

Together, the geographic distribution, activities of the vectors and reservoirs, and human activities determine the prevalence of rickettsial disease infections. In this study, $B$. indica appeared to be most influenced by $E$. chaffeensis (10.44\%), and $R$. tanezumi had the highest rate of seropositivity against $O$. tsutsugamushi $(23.08 \%)$. Although seropositive rates were highest in November and December for Ehrlichia spp., whereas the positive rates were higher in January and October for O. tsutsugamushi. The numbers of captured animals did not 
differ significantly between seasons, and the antibodies were detected year-round. In general, our results revealed that small mammals, which are common in residences, were infected by Ehrlichia and O. tsutsugamushi. The frequent association between these mammals and human activities may increase the risk of rickettsial diseases.

Our results indicated that the rates of seropositivity against $O$. tsutsugamush $i$ were higher in the eastern and central parts of Taiwan, which correlated with the high incidence of human scrub typhus in these regions [3]. Multiple environmental factors and vector species have been shown to affect the prevalence of vector-borne infectious pathogens in animal hosts [36-38]. A recent article has also reported a positive correlation between the rate of seropositivity in rodents and the incidence of human scrub typhus; however, the major captured animal in that study, S. murinus, was not tested due to a lack of antiserum [38]. FITC-conjugated goat anti-rat antibodies were used to detect antibodies from captured small mammals in our study. Similar strategies have been applied in other experiments when no antiserum was commercially available $[39,40]$. Although we cannot guarantee that anti-rat secondary antibodies react with rodent and shrew sera equally, the shrew was a minor species among all trapped small mammals (17.23\%). Zero and 4 (1.41 \%) shrews tested positive for antibodies against $E$. chaffeensis and $O$. tsutsugamushi, respectively.

Travel and trade have provided a source for disease dissemination throughout recorded history. Plague represents one of the most striking examples of this phenomenon. Human population movement has been crucial to the dispersal of Yersinia pestis and rodents and their fleas to new territories [41]. Moreover, international trade of pet reptiles and amphibians has been shown to have a potential to cause emerging infections of Rickettsia and Ehrlichia spp. in Japan [42]. In our study, animals were found to harbor antibodies against Ehrlichia and O. tsutsugamushi around ports and harbors. The prevalence of seropositivity was slightly higher in the samples collected near international harbors; however, no significant differences were observed. Nevertheless, serological evidence of infections was detected in all international harbors studied. These findings highlight the risk of spreading pathogenic Ehrlichia and Orientia associated with ectoparasites on rodents through aerial and marine transportation.

\section{Conclusions}

Our study provided serological evidence of Ehrlichia spp. infection in small mammals on the main island of Taiwan. The implications for human ehrlichiosis should be re-evaluated because it is not a commonly observed disease in Taiwan. Small mammals can serve as useful indicators and/or sentinels for epidemiological surveillance, disease control and prevention of vector-borne infectious diseases. The prevalence of infected animals captured around the harbors should raise awareness of the potential risks of disseminating pathogens by domestic and international aerial and marine transport.

\section{Abbreviations}

IFA: immunofluorescence assay; IgG: immunoglobulin G; PBS: phosphatebuffered saline.

\section{Competing interests}

The authors declare no conflicts of interest.

\section{Authors' contributions}

KHT and PYS participated in the design of the study and drafted the manuscript. SFC was involved in sample and antigen preparation and provided guidance for IFA. KHT and WJC performed IFAs. WLS and THW performed the statistical analyses and geographic mapping. XJY assisted in preparing the ehrlichial antigen. KHT, TYY, HCW, WJW and PYS reviewed the data and the manuscript. All authors critically reviewed and approved the final manuscript.

\section{Acknowledgements}

This research was supported by grants from the Ministry of Science and Technology (99-2314-B-002-126-MY3 and 103-2314-B002-036-MY2) and the Centers for Disease Control (DOH100-DC-2019). We thank Ms. Pei-Ru Wang for her technical assistance.

\section{Author details}

${ }^{1}$ Institute of Environmental Health, College of Public Health, National Taiwan University, No. 17, Xu-Zhou Road, Taipei 10055, Taiwan. ²Department of Public Health, College of Public Health, National Taiwan University, No. 17, Xu-Zhou Road, Taipei 10055, Taiwan. ${ }^{3}$ Center for Research, Diagnostics and Vaccine Development, Centers for Disease Control, Ministry of Health and Welfare, No.161, Kun-Yang Street, Taipei 11561, Taiwan. ${ }^{4}$ Institute of Epidemiology and Preventive Medicine, College of Public Health, National Taiwan University, No. 17, Xu-Zhou Road, Taipei 10055, Taiwan. ${ }^{5}$ Infectious Diseases Research and Education Center, Ministry of Health and Welfare and National Taiwan University, No. 17, Xu-Zhou Road, Taipei 10055, Taiwan. ${ }^{6}$ Departmentof Pathology, University of Texas Medical Branch, Galveston, TX, USA. ${ }^{7}$ Department of Geography, National Taiwan University, No. 1, Sec. 4, Roosevelt Road, Taipei 10617, Taiwan. ${ }^{8}$ Department of Entomology, College of Bioresources and Agriculture, National Taiwan University, No. 1, Sec. 4, Roosevelt Road, Taipei 10617, Taiwan.

Received: 17 November 2015 Accepted: 15 January 2016

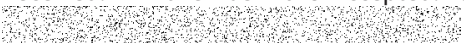

References

1. Tamura A, Ohashi N, Urakami H, Miyamura S. Classification of Rickettsia tsutsugamushi in a new genus, Orientia gen. nov., as Orientia tsutsugamushi comb. nov. Int J Syst Bacteriol. 1995;45(3):589-91.

2. Walker DH. Rickettsial diseases in travelers. Travel Med Infect Dis. 2003;1(1):35-40.

3. Lu HY, Tsai KH, Yu SK, Cheng CH, Yang JS, Su CL, et al. Phylogenetic analysis of 56-kDa type-specific antigen gene of Orientia tsutsugamushi isolates in Taiwan. Am J Trop Med Hyg. 2010;83(3):658-63.

4. Lin PR, Tsai HP, Tsui PY, Weng MH, Kuo MD, Lin HC, et al. Genetic typing, based on the 56-kilodalton type-specific antigen gene, of Orientia tsutsugamushi strains isolated from chiggers collected from wild-caught rodents in Taiwan. Appl Environ Microbiol. 2011;77(10):3398-405.

5. Wang $\mathrm{HC}$, Chung $\mathrm{CL}$, Lin $\mathrm{TH}$, Wang $\mathrm{CH}$, Wu WJ. Studies on the vectors and pathogens of scrub typhus on murine-like animals in Kinmen County, Taiwan. Formosa Entomol. 2004;24:257-72 (in Chinese).

6. Lin PR, Tsai HP, Weng MH, Lin HC, Chen KC, Kuo MD, et al. Field assessment of Orientia tsutsugamushi infection in small mammals and its association with the occurrence of human scrub typhus in Taiwan. Acta Trop. 2014;131:117-23.

7. Yabsley MJ. Natural history of Ehrlichia chaffeensis: vertebrate hosts and tick vectors from the United States and evidence for endemic transmission in other countries. Vet Parasitol. 2010;167:136-48. 
8. Dumler JS, Madigan JE, Pusterla N, Bakken JS. Ehrlichioses in humans: epidemiology, clinical presentation, diagnosis, and treatment. Clin Infect Dis. 2007;45 Suppl 1:S45-51.

9. Dumler JS, Barbet AF, Bekker CP, Dasch GA, Palmer GH, Ray SC, et al. Reorganization of genera in the families Rickettsiaceae and Anaplasmataceae in the order Rickettsiales: unification of some species of Ehrlichia with Anaplasma, Cowdria with Ehrlichia and Ehrlichia with Neorickettsia, descriptions of six new species combinations and designation of Ehrlichia equi and 'HGE agent' as subjective synonyms of Ehrlichia phagocytophila. Int J Syst Evol Microbiol. 2001;51(Pt 6):2145-65.

10. Cao WC, Gao YM, Zhang PH, Zhang XT, Dai QH, Dumler JS, et al. Identification of Ehrlichia chaffeensis by nested PCR in ticks from Southern China. J Clin Microbiol. 2000;38(7):2778-80.

11. Li HM, Jiang BG, He J, Niu JJ, Wang JX, Sun Y, et al. Detection and identification of Ehrlichia sp. in Boophilus microplus ticks from Xiamen of Fujian Province. J Patho Biol. 2006;1:174-6.

12. Weng MH, Lien JC, Tsai HP, Lin PR, Guo MD, Liu WT. Ehrlichia chaffeensis infection in rodent ticks - Kinmen, 2009. Taiwan. Taiwan Epi Bul. 2010;26:170-7.

13. Hsieh JW, Wang JT, Hiuang TM, Chen CH. Epidemiology investigation of rodents as vectors for the hantavirus in Taiwan's harbor area. Taiwan Epi Bull. 2008:24:68-87.

14. Weng MH, Tsai HP, Lin PR, Cheng KC, Guo MD, Liu WT. Investigation of Ehrlichia chaffeensis infections in rodents in Kinmen Area, 2012. Taiwan Epi Bull. 2014;30(7):134-41.

15. Yu XJ, Crocquet-Valdes PA, Cullman LC, Popov VL, Walker DH. Comparison of Ehrlichia chaffeensis recombinant proteins for serologic diagnosis of human monocytotropic ehrlichiosis. J Clin Microbiol. 1999;37(8):2568-75.

16. Tsai KH, Wang HC, Chen CH, Huang JH, Lu HY, Su CL, et al. Isolation and identification of a novel spotted fever group rickettsia, strain IG-1, from Ixodes granulatus ticks collected on Orchid Island (Lanyu), Taiwan. Am J Trop Med Hyg. 2008;79(2):256-61.

17. Magnarelli LA, Anderson JF, Stafford 3rd KC, Dumler JS. Antibodies to multiple tick-borne pathogens of babesiosis, ehrlichiosis, and Lyme borreliosis in white-footed mice. J Wildl Dis. 1997;33(3):466-73.

18. Marshall GS, Jacobs RF, Schutze GE, Paxton H, Buckingham SC, DeVincenzo JP, et al. Ehrlichia chaffeensis seroprevalence among children in the southeast and south-central regions of the United States. Arch Pediatr Adolesc Med. 2002;156(2):166-70

19. Paddock CD, Childs JE. Ehrlichia chaffeensis: a prototypical emerging pathogen. Clin Microbiol Rev. 2003;16(1):37-64.

20. Dugan VG, Little SE, Stallknecht DE, Beall AD. Natural infection of domestic goats with Ehrlichia chaffeensis. J Clin Microbiol. 2000;38(1):448-9.

21. Dugan VG, Gaydos JK, Stallknecht DE, Little SE, Beall AD, Mead DG, et al. Detection of Ehrlichia spp. in raccoons (Procyon lotor) from Georgia. Vector Borne Zoonotic Dis. 2005;5(2):162-71.

22. Kocan AA, Levesque GC, Whitworth LC, Murphy GL, Ewing SA, Barker RW. Naturally occurring Ehrlichia chaffeensis infection in coyotes from Oklahoma. Emerg Infect Dis. 2000;6(5):477-80.

23. Davidson WR, Lockhart JM, Stallknecht DE, Howerth EW. Susceptibility of red and gray foxes to infection by Ehrlichia chaffeensis. J Wildl Dis. 1999;35(4):696-702.

24. Wen B, Cao W, Pan H. Ehrlichiae and ehrlichial diseases in China. Ann NY Acad Sci. 2003;990:45-53.

25. Lockhart JM, Davidson WR, Stallknecht DE, Dawson JE, Little SE. Natural history of Ehrlichia chaffeensis (Rickettsiales: Ehrlichieae) in the piedmont physiographic province of Georgia. J Parasitol. 1997;83(5):887-94.

26. Chien $\mathrm{CH}$, Chiang PF, Wang HC, Chen KY, Lin MC, Wu HS. Prevalence of ectoparasites and the seroepidemiology of murine typhus in murine-like animals at international ports in Taiwan, 2004-2011. Taiwan Epidemiol Bull. 2012;28(22):320-9.

27. Maeda K, Markowitz N, Hawley RC, Ristic M, Cox D, McDade JE. Human infection with Ehrlichia canis, a leukocytic rickettsia. N Engl J Med. 1987; 316(14):853-6.

28. Kelly PJ, Matthewman LA, Mahan SM, Semu S, Peter T, Mason PR, et al. Serological evidence for antigenic relationships between Ehrlichia canis and Cowdria ruminantium. Res Vet Sci. 1994;56(2):170-4.

29. Buller RS, Arens M, Hmiel SP, Paddock CD, Sumner JW, Rikhisa Y, et al. Ehrlichia ewingii, a newly recognized agent of human ehrlichiosis. N Engl J Med. 1999;341(3):148-55

30. Jongejan F, de Vries N, Nieuwenhuijs J, Van Vliet AH, Wassink LA. The immunodominant 32-kilodalton protein of Cowdria ruminantium is conserved within the genus Ehrlichia. Rev Elev Med Vet Pays Trop. 1993; 46(1-2):145-52.

31. Huang CC, Hsieh YC, Tsang CL, Chung YT. Sequence and phylogenetic analysis of the gp200 protein of Ehrlichia canis from dogs in Taiwan. J Vet Sci. 2010;11(4):333-40.

32. Yuasa Y, Hsu TH, Chou CC, Huang CC, Huang WC, Chang CC. The comparison of spatial variation and risk factors between mosquito-borne and tick-borne diseases: Seroepidemiology of Ehrlichia canis, Anaplasma species, and Dirofilaria immitis in dogs. Comp Immunol Microbiol Infect Dis. 2012;35(6):599-606.

33. Beall MJ, Alleman AR, Breitschwerdt EB, Cohn LA, Couto CG, Dryden MW, et al. Seroprevalence of Ehrlichia canis, Ehrlichia chaffeensis and Ehrlichia ewingii in dogs in North America. Parasit Vectors. 2012;5:29.

34. Zhang L, Liu H, Xu B, Zhang Z, Jin Y, Li W, et al. Rural residents in China are at increased risk of exposure to tick-borne pathogens Anaplasma phagocytophilum and Ehrlichia chaffeensis. BioMed Res Int. 2014;2014(2014):1-11.

35. Kuo CC, Huang CL, Wang HC. Identification of potential hosts and vectors of scrub typhus and tick-borne spotted fever group rickettsiae in eastern Taiwan. Med Vet Entomol. 2011;25(2):169-77.

36. Cooper WC, Lien JC, Hsu SH, Chen WF. Scrub typhus in the Pescadores Islands: An epidemiologic and clinical study. Am J Trop Med Hyg. 1964;13:833-8.

37. Lee YS, Wang PH, Tseng SJ, Ko CF, Teng HJ. Epidemiology of scrub typhus in eastern Taiwan, 2000-2004. Jpn J Infect Dis. 2006;59(4):235-8.

38. Kuo CC, Huang JL, Ko CY, Lee PF, Wang HC. Spatial analysis of scrub typhus infection and its association with environmental and socioeconomic factors in Taiwan. Acta Tropica. 2011;120:52-8.

39. Luan VD, Yoshimatsu K, Endo R, Taruishi M, Huong VT, Dat DT, et al. Studies on hantavirus infection in small mammals captured in southern and central highland area of Vietnam. J Vet Med Sci. 2012;74(9):1155-62.

40. Guan D, Li W, Su J, Fang L, Takeda N, Wakita T, et al. Asian musk shrew as a reservoir of rat hepatitis E virus, China. Emerg Infect Dis. 2013;19(8):1341-3.

41. Wilson ME. Travel and the emergence of infectious diseases. Emerg Infect Dis. 1995;1 (2):39-46.

42. Andoh M, Sakata A, Takano A, Kawabata H, Fujita H, Une Y, et al. Detection of Rickettsia and Ehrlichia spp. In ticks associated with exotic reptiles and amphibians imported into Japan. PLoS ONE. 2015;10(7):e0133700.

\section{Submit your next manuscript to BioMed Central and we will help you at every step:}

- We accept pre-submission inquiries

- Our selector tool helps you to find the most relevant journal

- We provide round the clock customer support

- Convenient online submission

- Thorough peer review

- Inclusion in PubMed and all major indexing services

- Maximum visibility for your research

Submit your manuscript at www.biomedcentral.com/submit
) Biomed Central 